\title{
Survey of neonatal respiratory support use in very preterm infants in Italy
}

\author{
Luigi Gagliardi ${ }^{1}$, Paolo Tagliabue ${ }^{2}$, Roberto Bellù ${ }^{3}$, Carlo Corchia ${ }^{4}$, Fabio Mosca ${ }^{5}$, Rinaldo Zanini ${ }^{3}$, for the \\ Network Neonatale Italiano
}

\begin{abstract}
${ }^{1}$ Pediatrics and Neonatology Division, Woman and Child Health Department, Ospedale Versilia, Lido di Camaiore, Italy, ${ }^{2}$ Neonatology and Neonatal Intensive Care Unit, Fondazione MBBM - Ospedale San Gerardo Monza, Italy, ${ }^{3}$ Neonatal Intensive Care Unit, Ospedale "A Manzoni", Lecco, Italy, ${ }^{4}$ International Centre on Birth Defects and Prematurity, Rome, Italy, and ${ }^{5}$ Department of Maternal and Pediatric Sciences, University of Milan; Fondazione IRCCS Cà Granda Ospedale Maggiore Policlinico, Milan, Italy
\end{abstract}

Background: Respiratory support in very preterm infants is often a life-saving procedure and several techniques are available. There is lack of data on the current use of these techniques. Methods and aim: We analyzed a cohort of infants $<30$ weeks gestation or $<1501 \mathrm{~g}$ birth weight, enrolled in the Italian Neonatal Network in 2009 and 2010 ( $n=8297$, mean gestation $=29.3$ weeks, mean birth weight $=1089 \mathrm{~g}$ ) to ascertain the use of several techniques. We also conducted a questionnaire survey of all neonatal units adhering to the Italian Neonatal Network, inquiring about preferred methods of respiratory support. Results: Conventional ventilation was used in $53 \%$ of these infants, high frequency ventilation in $15.8 \%$, CPAP in $71.6 \%$, and surfactant in $56.2 \%$. Huge variations were observed between hospitals for all techniques studied. The survey of centres showed that all respondents considered non-invasive ventilation the first-intention treatment for these infants. In case of need of tracheal intubation and mechanical ventilation, two hospitals said they used IPPV/ IMV; 20 synchronized IPPV/IMV; 25 "volume guarantee"; and 10 hospitals used HFV as a first intention treatment (in five hospital for all infants, and in other five hospitals, depending upon birth weight/gestation. Conclusions: This study provides large scale, up to date results of the current methods of respiratory support in very preterm infants in Italy. Huge variations between hospitals, only partly explained by the paucity of evidence-based data, are apparent. These differences point to the possibility of implementing "potentially better practices" with the aim of reducing unwanted, physician-related variation.

Keywords: Ventilation, continuous positive airway pressure, variation, preterm infant, respiratory management

Respiratory support in very preterm infants is often a life-saving procedure. In the last several years, a great number of techniques have been proposed and used to treat respiratory insufficiency in neonates, including both invasive (e.g. "conventional" mechanical ventilation, both synchronized and non-synchronized, with and without volume guarantee; time-cycled and flow-cycled or high frequency ventilation $[\mathrm{HFV}]$ ) and "non-invasive" (e.g. nasal continuous positive airway pressure (CPAP), nasal intermittent mandatory ventilation, with high flow nasal cannulae) techniques.

Most of the evidence regarding differences between different techniques stems from small studies, often with short term outcomes. On the contrary, large randomized controlled trials and systematic reviews mostly prove equivalence in short and long term outcomes among the newborns treated with different respiratory assistance techniques [1-5].

Ideally, the type of respiratory support should match the degree of respiratory compromise, with a gradient from lesser to greater invasiveness, but there is large room for individual opinions in the matter of ventilation/respiratory support for the preterm infant. As a consequence, the "market share" of each technique is unknown, and there are very little epidemiological data available from scientific literature.

One way to approach this problem is asking directors of clinical units about their "standard" management of preterm infants. A famous example is the "eight centres" survey that put emphasis on CPAP [6]; some years ago, Sharma and Greenough [7] conducted a survey among UK chiefs of neonatal units about their preferred management. These surveys can be extremely useful in understanding what the opinions of senior clinicians/ opinion leaders are, but they do not report data on the current methods of providing respiratory support in neonates.

In literature there are no suitable, recent estimates of how these various techniques are currently used in neonatal intensive care units (NICUs). The availability of data from large neonatal networks allows an objective assessment of use of various techniques.

Given the high impact of respiratory techniques in neonatology, and the lack of information about their use, to learn more objective data about current respiratory management of very preterm infants, we adopted a dual strategy: (1) an analysis of the data of the respiratory support offered to individual very preterm neonates enrolled in the Network Neonatale Italiano (Italian Neonatal Network, INN), and (2) a brief survey of the protocols in use in the neonatal units adhering to the INN.

The aim of this study is to describe the current respiratory management of very preterm neonates in Italy.

\section{Methods}

\section{INN database analysis}

We analyzed the dataset of infants enrolled in the INN in 2009 and 2010. The INN is the Italian branch of the Vermont-Oxford Network (VON). It includes preterm neonates up to 29 weeks +6 
days, or neonates with a birth weight $<1501 \mathrm{~g}$, admitted to any of the centres within 1 month of age.

In 2009, 73 centres participated, and in 2010 a further 10 contributed data (Total $=83$ ). Although the exact number of NICUs in Italy is not known, about 110 centres are believed to be a correct estimate. Thus, the neonatal units participating in the INN care vast majority of very preterm infants in Italy, and in some Italian regions the coverage is complete. Therefore, the INN represents an important source of data that reflect neonatal management in Italy.

All definitions are those of the VON (www.vtoxford.org); briefly, in addition to anthropometric measures such as gestational age (GA), birth weight (BW), sex, etc, the following variables are collected for each infant: respiratory management in the delivery room (use of oxygen, bag-and-mask ventilation, intubation, surfactant administration) and in the ward. As far as ventilatory support is concerned, the database collects data on procedures such as oxygen supplementation; use of CPAP, of CPAP before intubation; of high flow nasal cannulae; of nasal intermittent mandatory ventilation (IMV)/synchronized intermittent mandatory ventilation (SIMV); of conventional ventilation; of HFV; and surfactant administration in the ward. Bronchopulmonary dysplasia (BPD) refers to the oxygen requirement at 36 weeks.

The data of the INN is compared with the data from the whole VON in 2010 (more than 57000 infants with the same characteristics), obtained from the web-based system "Nightingale".

\section{Questionnaire analysis}

The questionnaire (available on request) was prepared by PT and sent by email to all chiefs of neonatal units adhering to the INN, with reminders in case of non-response. It included questions on the existence in the unit of protocols detailing the approach to respiratory diseases in the neonates, and which type of respiratory support was chosen as first line therapy and whether HFV was used for rescue in the event of a failure of conventional ventilation. Fifty-seven hospitals (69\%) responded.

\section{Results}

The study included 8297 infants (3981 in 2009, and 4316 in 2010), treated in 83 hospitals. For the purpose of this study, we only excluded infants dying in the delivery room $(n=73)$.

The mean GA was 29.3 weeks (median $=29.4, \mathrm{SD}=2.96)$ and the mean BW was $1089 \mathrm{~g}$ (median $=1130, \mathrm{SD}=302)$.

Mortality was $14.2 \%$, and BPD (supplemental oxygen at 36 weeks postmenstrual age) $13.9 \%$ ( $15.5 \%$ in infants $<33$ weeks).

Table I shows the percentage of infants receiving any of the listed procedures, comparing the data with the data from the VON. The frequency of use of most procedures is lower in Italian hospitals in comparison to the VON.

Given that INN recruits infants above 30 weeks GA only if they are below $1501 \mathrm{~g} \mathrm{BW}$, with an increase of small for gestational age (SGA) infants with increasing GA, these procedures are displayed in Table II for infants of 23-29 weeks only (the data for this population is complete in the database). The GA-associated decrease in the use of procedures is clearly apparent.

Table III provides details regarding a particular procedure are shown; i.e. a comparison in the time to surfactant administration in infants of 23-29 weeks between INN and rest of the VON. For all GA weeks considered, the percentage of infants treated with surfactant $>2 \mathrm{~h}$ of age is always greater in Italy than in the VON, even for very preterm infants, where surfactant is almost universally administered (see Table II).
Table I. Percentage of infants receiving any of the listed procedures.

\begin{tabular}{lrr}
\hline & INN & VON \\
\hline Delivery room procedures & & \\
Supplemental oxygen & 76.29 & 85.3 \\
Bag-and-mask ventilation & 61.39 & 61.8 \\
Tracheal intubation & 41.49 & 50.8 \\
Surfactant administration & 16.80 & 32.3 \\
After delivery room & & \\
Oxygen & 80.80 & 88.2 \\
Conventional ventilation & 53.19 & 62.7 \\
HFV & 15.84 & 22.1 \\
nCPAP & 71.57 & 68.2 \\
CPAP before ETT & 41.99 & 40.0 \\
Any surfactant & 56.18 & 63.1 \\
HFNC & 8.26 & 49.9 \\
NIMV/NSIMV & 17.66 & 18.7 \\
Any mechanical ventilation & 55.56 & 65.1 \\
Non-invasive ventilation & 71.73 & - \\
Any respiratory assistance & 84.53 & - \\
NIV only & 34.02 & - \\
\hline
\end{tabular}

INN, Italian Neonatal Network; VON, Vermont-Oxford Network, 2010; ETT, endotracheal intubation; HFNC, high flow nasal cannula; NIMV, nasal intermittent mechanical ventilation; NIV, non-invasive ventilation.

aVentilation or NIV, excluding oxygen supplementation.

bIn infants receiving any respiratory assistance, excluding oxygen supplementation.

Table II. Percentage of use of selected methods of respiratory support according to GA, 23-29 weeks (percentage).

\begin{tabular}{lcrrrrrrr}
\hline & \multicolumn{7}{c}{ Gestational age (weeks) } & \\
\cline { 2 - 8 } & \multicolumn{1}{c}{23} & \multicolumn{1}{c}{24} & \multicolumn{1}{c}{25} & 26 & \multicolumn{1}{c}{27} & 28 & 29 & Total \\
\hline DR surfactant & 51.88 & 41.82 & 42.68 & 34.87 & 27.64 & 18.25 & 11.32 & 26.96 \\
Any Surfactant & 96.62 & 98.18 & 94.35 & 89.06 & 8 & 74.27 & 61.97 & 80.06 \\
MV & 99.63 & 99.48 & 96.05 & 90.68 & 80.85 & 67.40 & 53.08 & 76.60 \\
NIV & 36.43 & 56.92 & 70.83 & 83.65 & 87.78 & 89.08 & 87.26 & 80.01 \\
Any Resp. assis. & 99.63 & 100.00 & 99.58 & 99.68 & 99.58 & 97.48 & 93.73 & 97.70 \\
NIV only & 0.00 & 0.52 & 3.55 & 9.03 & 18.76 & 30.79 & 42.91 & 21.52 \\
\hline
\end{tabular}

$\mathrm{DR}$, delivery room; $\mathrm{MV}$, mechanical ventilation; NIV, non-invasive ventilation; NIV only, NIV without need for MV.

Table III. Time to surfactant administration $>2$ h: comparison between INN and VON (percentage of infants).

\begin{tabular}{lll}
\hline Gestational age (weeks) & INN & VON \\
\hline 23 & 10.55 & 4.90 \\
24 & 14.63 & 6.30 \\
25 & 16.78 & 7.40 \\
26 & 17.32 & 10.00 \\
27 & 32.58 & 14.10 \\
28 & 37.48 & 18.50 \\
29 & 46.88 & 24.40 \\
\hline
\end{tabular}

INN, Italian Neonatal Network; VON, Vermont-Oxford Network, 2010.

Figures on the average use of various techniques even when split in weeks of GA, tell only part of the story, because variation across centres is conspicuous.

For six selected procedures (Delivery room intubation; delivery room surfactant; any mechanical ventilation, HFV, CPAP before intubation, non-invasive ventilation), Figure 1 provides the mean use of each procedure for each of the 83 hospitals. The variation is very great for all procedures, indicating that the mean value reported in Tables I-III for all procedures is, in reality, an abstraction. Both 

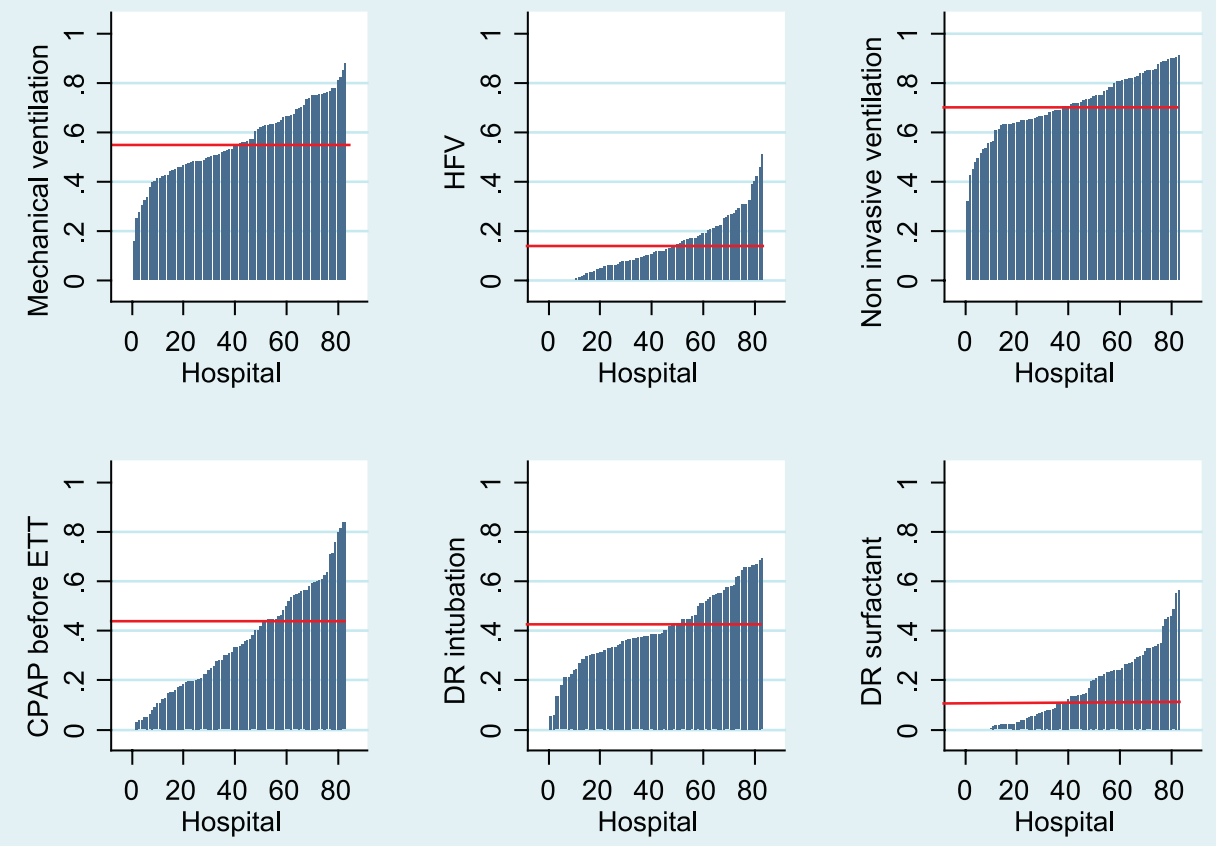

Figure 1. Variation in the mean percentage of use of the various techniques across 83 hospitals, ranked in ascending order of use of each technique. Each bar represents a hospital. The red line represents the mean for all hospitals.

Table IV. Correlations between mean use of selected procedures between hospitals.

\begin{tabular}{lrrrrrr}
\hline & DRET & DR SURF & CPAPES & HFV & VENT & NIV \\
\hline DRET & 1.0000 & & & & & \\
DR surfactant & 0.3678 & 1.0000 & & & & \\
CPAPES & -0.4494 & -0.0817 & 1.0000 & & & \\
HFV & 0.3101 & -0.0069 & 0.1039 & 1.0000 & & \\
Vent & 0.6499 & 0.1517 & -0.5280 & 0.0177 & 1.0000 & \\
NIV & -0.1053 & -0.0564 & 0.3298 & 0.0816 & -0.1604 & 1.0000 \\
NIV only & -0.6412 & -0.1520 & 0.5638 & -0.0915 & -0.8845 & 0.4752 \\
\hline
\end{tabular}

DR, delivery room; HFV, high frequency ventilation; NIV, non-invasive ventilation; NIV only, NIV without need for mechanical ventilation. CPAPES, CPAP before ventilation; DRET, delivery room intubation

the pattern, and the extent of variation differs across the procedures: the coefficient of variation is lower for non-invasive ventilation (0.17), ventilation (0.26) and delivery room intubation (0.36), than for CPAP before endotracheal intubation (ETT) (0.58), and is highest for HFV (0.86) and delivery room surfactant (0.89).

Interestingly, the correlation between the mean use of techniques across hospitals (Table IV) shows that some techniques are not very correlated with one other. For instance, HFV is only associated with delivery room (DR) intubation $(r=0.31)$, but not with conventional ventilation $(r=0.02)$, i.e. hospitals that use more HFV do not use less conventional ventilation. NIV-only is negatively affected (as expected) by conventional ventilation (i.e. hospitals that ventilate more, use less NIV-only), but not with HFV (hospitals that use more HFV have the same number of infants managed non-invasively).

Considered together, this data suggest that it is difficult to ascribe this broad variation to differences in case-mix, and it likely represents a "physician-driven" variation.

As for the results of the questionnaire, of the 57 hospitals that responded to the questionnaire, six said they did not have a protocol for respiratory assistance and were excluded from further analysis; all the others said that they used "non-invasive" techniques first.

In the event of needing tracheal intubation and mechanical ventilation as a first-intention treatment, two hospitals said they used IPPV/IMV; 20 synchronized IPPV/IMV; 25 "volume guarantee"; and 10 hospitals used HFV (in five hospital for all infants, and in other five hospitals, depending upon BW/GA). (The figures do not add up to 51 because in some cases hospitals had a dual first choice).

As for rescue HFV, seven hospitals (among those that did not always use HFV as a first intention) responded that they did not use HFV as a rescue, while all the others did use HFV as rescue.

\section{Discussion}

To the best of our knowledge, this study represents the first large scale, current survey of actual techniques used to provide respiratory assistance to very preterm infants, admitted to intensive/ special care baby units in Italy in very recent years.

In fact, our conclusions rest on more than 8000 very preterm/ very small neonates assisted in 83 centres in 2009-2010.

This data confirms that ventilatory assistance remains one of the most important and most used procedures for these infants. Focusing only on infants of 23-29 weeks demonstrates that, as expected, the number of infants receiving each treatment decreases with the GA. Of great interest, in light of the current trend towards a lower level of invasiveness, is the number of infants who are managed without "invasive" (i.e. without endotracheal tube positioning) techniques. While invasive ventilation is always used at the lower extreme of viability, our data show that at 29 weeks, only half of the infants require intubation and receive at least one dose of surfactant, while the other half is managed non-invasively. The comparison with the data of VON shows that, on the whole, Italian neonates are treated less invasively. 
In addition to the current description of use of various techniques, this study shows great variations in their use. This variation has been frequently reported [8-12] in studies on ventilation.

Of note, most of the procedures surveyed and employed (CPAP, mechanical ventilation, even HFV), can nowadays be considered "standard", and date back several years, so that these differences between hospitals cannot be due to a novelty of the idea of various techniques. Despite the long time that HFV, conventional mechanical ventilation, and nasal continuous positive airway pressure (NCPAP) have been available, the differences between the centres are striking, and probably reflect different approaches to the preterm infants with respiratory distress, i.e. physician-driven variation, rather than differences in case-mix.

The lack of solid data to guide therapeutic decisions is certainly an important cause of this variation [1-5]. However, also the lack of an attitude towards evidence-based approach to ventilation plays an important role.

We believe that the reason for these discrepancies is that the practice of neonatology has a large "handicraft" (as opposed to "engineered", scientific) component. In this scenario, different neonatal units put more emphasis on locally-developed protocols and "mindlines", building on historical experience developed over years (a "school" of thought) rather than trying to conform to objective recommendations. A telling example is the difference between INN and VON for the timing of surfactant administration. For very low GA infants at 24-26 weeks GA, surfactant is almost always used. Meta-analyses recommend prophylactic administration over rescue administration $[13,14]$, and it is likely that by $2 \mathrm{~h}$ of age, such very small infants have "declared" themselves (i.e. those with a respiratory distress syndrome (RDS) requiring surfactant are recognizable). Yet, $14 \%$ of infants at 24 weeks GA are treated with surfactant beyond $2 \mathrm{~h}$ of age. Another example is that the number of units using first-intention HFV (and the number of infants treated with HFV) is much smaller than what one would expect knowing that - if anything - HFV is equal or better than "conventional" ventilation for preterm infants $[4,5]$.

The survey conducted among chiefs of neonatal units helps us supplement the analysis of infants. In fact, it demonstrates that none of the respondents considers NIV unsuitable for a trial in infants. In practice, however, we can see that CPAP before intubation is used from $0 \%$ to about $80 \%$ of infants. As for the choice of invasive (with intubation) ventilation, only two hospitals used a non-synchronized "conventional" ventilation, five used HFV always, and the other five used HFV in some (smaller/ more immature) infants only. The vast majority of units used synchronized ventilation, with or without volume guarantee. In a survey carried out in the UK and published in 2007 [7], only 2\% of units chose CPAP as initial treatment of acute RDS, $2 \%$ used HFV, $22 \%$ used some form of synchronized ventilation, while non-synchronized conventional ventilation was chosen by $73 \%$. The differences between years and between countries may explain these discrepancies.

It is, however, tempting to speculate that the low frequency of BPD observed in these infants is at least partly due to less invasive techniques.

This study had the obvious limitation that the VON (and INN) database does not include details about the duration of support, nor does it enable us to ascertain the time course of events (e.g. when ventilation is started and stopped, or what techniques are used after extubation), so neither the amount of resources absorbed nor the actual clinical trajectories of infants are known.
These data, however, form a basis for recognizing areas for potential improvement in ventilation given to preterm neonates. The great variation across hospitals in all the surveyed techniques points to the possibility of implementing "potentially better practices" (such as the widespread use of delivery room CPAP or gentle ventilation; reduced intubation at birth; greater use of HFV or low tidal volume ventilation, more consistent use of prophylactic surfactant in very tiny infants, etc.) with the aim of reducing unwanted, physician-related variation. The use of data impressively shows the power of large neonatal networks in identifying areas for potential improvement.

Declaration of Interest: The Italian Neonatal Network is supported by an unrestricted grant by Chiesi Pharmaceuticals. The authors report no declaration of interest.

\section{References}

1. Morley CJ, Davis PG, Doyle LW, Brion LP, Hascoet JM, Carlin JB; COIN Trial Investigators. Nasal CPAP or intubation at birth for very preterm infants. N Engl J Med 2008;358:700-708.

2. Greenough A, Dimitriou G, Prendergast M, Milner AD. Synchronized mechanical ventilation for respiratory support in newborn infants. Cohrane review http://www.nichd.nih.gov/cochrane/.

3. Sandri F, Plavka R, Ancora G, Simeoni U, Stranak Z, Martinelli S, Mosca F, et al.; CURPAP Study Group. Prophylactic or early selective surfactant combined with nCPAP in very preterm infants. Pediatrics 2010;125:e1402-e1409.

4. Cools F, Henderson-Smart DJ, Offringa M, Askie LM. Elective high frequency oscillatory ventilation versus conventional ventilation for acute pulmonary dysfunction in preterm infants. Cochrane Database Syst Rev 2009;CD000104.

5. Cools F, Askie LM, Offringa M, Asselin JM, Calvert SA, Courtney SE, Dani C, et al.; PreVILIG collaboration. Elective high-frequency oscillatory versus conventional ventilation in preterm infants: a systematic review and meta-analysis of individual patients' data. Lancet 2010;375:2082-2091.

6. Avery ME, Tooley WH, Keller JB, Hurd SS, Bryan MH, Cotton RB, Epstein MF, et al. Is chronic lung disease in low birth weight infants preventable? A survey of eight centers. Pediatrics 1987;79:26-30.

7. Sharma A, Greenough A. Survey of neonatal respiratory support strategies. Acta Paediatr 2007;96:1115-1117.

8. Horbar JD, McAuliffe TL, Adler SM, Albersheim S, Cassady G, Edwards W, Jones R, et al. Variability in 28-day outcomes for very low birth weight infants: an analysis of 11 neonatal intensive care units. Pediatrics 1988;82:554-559.

9. Van Marter LJ, Allred EN, Pagano M, Sanocka U, Parad R, Moore M, Susser M, et al. Do clinical markers of barotrauma and oxygen toxicity explain interhospital variation in rates of chronic lung disease? The Neonatology Committee for the Developmental Network. Pediatrics 2000;105:1194-1201.

10. Lee SK, McMillan DD, Ohlsson A, Pendray M, Synnes A, Whyte R, Chien LY, Sale J. Variations in practice and outcomes in the Canadian NICU network: 1996-1997. Pediatrics 2000;106:1070-1079.

11. Vohr BR, Wright LL, Dusick AM, Perritt R, Poole WK, Tyson JE, Steichen JJ, et al.; Neonatal Research Network. Center differences and outcomes of extremely low birth weight infants. Pediatrics 2004;113:781-789.

12. Gagliardi L, Bellù R, Lista G, Zanini R; Network Neonatale Lombardo Study Group. Do differences in delivery room intubation explain different rates of bronchopulmonary dysplasia between hospitals? Arch Dis Child Fetal Neonatal Ed 2011;96:F30-F35.

13. Engle WA; American Academy of Pediatrics Committee on Fetus and Newborn. Surfactant-replacement therapy for respiratory distress in the preterm and term neonate. Pediatrics 2008;121:419-432.

14. Stevens TP, Harrington EW, Blennow M, Soll RF. Early surfactant administration with brief ventilation vs. selective surfactant and continued mechanical ventilation for preterm infants with or at risk for respiratory distress syndrome. Cohrane review http://www.nichd.nih. gov/cochrane/. 


\section{Appendix: Participants to the Network Neonatale Italiano}

Lecco (Gianpaolo Mirri, Manuela Condò, D Turoli); Milano Mangiagalli (Gaia Weissmann, Mara Vanzati); Trento (Giuseppe De Nisi); Como S. Anna (E Villa); Milano Macedonio Melloni (Guido Moro); Ferrara (Pietro Guerrini, Raffaella Contiero); Udine (Serena Ellero, Riccardo Furlan); Monza (Laura Abbiati, Cesarina Borroni); Torino S.Anna (Giovanna Prandi, Claudio Fabris, Francesca Vielmi); Varese (Massimo Agosti, Francesco Tandoi, Guidali); Roma. Umberto I (Mario De Curtis, Claudio Tozzi, Renato Lucchini); Milano Buzzi (Marina Battaglioli, Gian Luca Lista, Paola Introvini); Modena. (Fabrizio Ferrari, Claudio Gallo); Palermo Cervello (Elvira Bellante); Mantova (Chiara Bottura, Francesca Pasquali, Simona Boccacci); Brindisi (Giuseppe Latini, Raffaella Giannuzzi); Milano Niguarda (Stefano Martinelli, Alberto Brunelli, Giuliana Motta); Campobasso (Maria Lucia Di Nunzio, Antonio Vendemiati); Camposampiero (Carlo Zorzi, Giovanna Carli, Alfiero); Rimini (Nicola Romeo, Palma Mammoliti); Torino (Emanuele Mastretta, Laura Barberis, Daniele Farina); Cuneo (Gianpaolo Gancia, Cristina Dalmazzo); Napoli Villa Betania(Marcello Napolitano, Francesco Messina); Foggia. (Giuseppe Rinaldi, Rosario Magaldi, Matteo Rinaldi, Rosangela Litta); Padova (Paola Lago, Vincenzo Zanardo, Lino Chiandetti); Treviso (Stefano Visentin); Tricase (Giuseppe Presta, Davide Cella); Cremona (Carlo Poggiani, Daniela Ferrari, Sara Parati); Siracusa (Francesco Lombardo, Rosetta Grigorio); Milano San Raffaele (Graziano Barera, Margherita Franco, Antonella Poloniato); Bergamo (Angelo Colombo, Giampiero Burgio,Elena Sala); Messina (Ignazio Barberi, Venera Tiralongo, Alessandro Arco); Firenze Careggi (Carlo Dani, Simone Pratesi); Bologna S.Orsola (Valeria Mignatti, Gina Ancora, Giacomo Faldella, Sara Grandi); Pavia (Mauro Stronati, Gianfranco Perotti); Brescia (Gaetano Chirico, Claudio Migliori); Trieste (Sergio De Marini, Vincenzo Forleo); Napoli Federico II (Roberto Paludetto, Letizia Capasso, Giuseppina Mansi, Francesco Raimondi); Novara (Gianni Bona, Ilaria Stucchi, Savastio, Federica Ferrero, Andrea Parola); Verona Borgo Trento (Ezio Maria Padovani, Elena Viviani, Laura Pecoraro); Roma. Fatebenefratelli Isola Tiberina (Rocco Agostino, Camilla Gizzi, Luca Massenzi); Bolzano (Hubert Messner, Alex Staffler); Napoli Buon Consiglio - F.B.F. (Gennaro Salvia); Acquaviva (Luigi Esposito, Vincenzo Forziati, Giuseppe
Latorre); Bologna Ospedale Maggiore (Fabrizio Sandri, Stefania Alati, Fabrizio Demaria); Cosenza (Oreste Lombardi, Carmine Deni Costabile, Gianfranco Scarpelli); Parma (Claudio Cavalli, Enrico Volante, Dott.ssa Proietti); Palermo Buccheri La Ferla F.B.F. (Olga Ganguzza, Bartolomeo Spinella); Roma S. Pietro F.B.F. (Cristina Haass, Eleonora Scapillati, Chiara Consigli); S.Giovanni Rotondo (Alberto Gatta, Pasqua Quitadamo); Pisa (Antonio Boldrini, Marco Vuerich, Emilio Sigali, Paolo Ghirri); Firenze Meyer (Patrizio Fiorini, Letizia Petrucci, Marco Moroni); Perugia (Patrizia Braghetti, Paola Casucci, Liliana Minelli, Daniele Mezzetti); Benevento (Luigi Orfeo, Maria Gabriella De Luca); Bari Policlinico (Nicola Laforgia, Alessandro Grassi); Roma Bambino Gesù (Andrea Dotta, Ferdinando Savignoni); Siena (Franco Bagnoli, Claudio De Felice, Silvia Badii); Cesena (Augusto Biasini, Antonio Belluzzi, Marcello Stella); Roma Gemelli (Costantino Romagnoli, Enrico Zecca, Giovanni Barone); Vicenza (Paolo Colleselli, Luca Vecchiato, Dott.ssa Nicolussi); Napoli Monaldi (Paolo Giliberti, Giovanni Chello, Silvana Rojo); Rho (Chiara Giovanettoni, Clelia A. Colnaghi); Versilia (Elena Verucci, Giulia Placidi); Lodi (Cesare Belloni, Giuseppe Carrera, Chiara Zambetti); Verona Ospedale Civile Maggiore (Paolo Biban, Alessandra Serra); Ancona (Virgilio Carnielli, Annalisa Pedini); Benevento Ospedale Fatebenefratelli (Gennaro Vetrano, Giuseppe Furcolo, Brigida Pasquariello); Caserta (Luigi Falco, Gaetano Ausanio, Italo Bernardo); Nocera Inferiore (Giuseppe Marchesano, Norberto Nosari, Paola Sarnelli); Como Valduce (Mario Maccabruni, Daniele Merazzi); Alessandria (Diego Gazzolo, Francesca Temporini Miriam Sabatini, Micaela Colivicchi); Bari Di Venere (Antonio Del Vecchio, Marzia Tarantino); Reggio Emilia (Giancarlo Gargano, Dsimona Pedori); Thiene (Massimo Bellettato, Roberta Pesavento, Alessandra Cesaro); Reggio Calabria (Antonio Nicolò, Isabella Mondello); Napoli Cardarelli (Aniello Pugliese, Claudio Iervolino); Palermo Policlinico Giaccone (Giovanni Corsello, Mario Giuffré); Catania Policlinico Rodoligo, Catania (Maria Betta, Mario Giuseppe Romeo, Alessandro Saporito); Pescara (Carmine D'Incecco, Barbara Perrone); Catania. Ospedale Santo Bambino (Maria Grazia Leone Alessandro Rodonò); Genova Gaslini (Giovanni Serra, Alessia Franceschi, Francesco M. Risso); Napoli SS Annunziata (Mauro Carpentieri, Maria Pia Cigliano); Roma Casilino (Piermichele Paolillo, Simonetta Picone); Moncalieri (Antonio Marra, Dott.ssa Rossetti, Tiziana Testa); Lecce (Fernando Del Cuore). 